\title{
Impact of maternal dietary fatty acid composition on glucose and lipid metabolism in male rat offspring aged $105 \mathrm{~d}$
}

\author{
Ahamed Ibrahim, Ghafoorunissa*, Sanjay Basak and Nasreen Z. Ehtesham \\ Department of Biochemistry, National Institute of Nutrition, Hyderabad 500 007, India
}

(Received 16 April 2008 - Revised 4 November 2008 - Accepted 7 November 2008 - First published online 23 January 2009)

In recent years the intake of $n$ - 6 PUFA and trans-fatty acids (TFA) has increased, whereas $n$ - 3 PUFA intake has decreased. The present study investigated the effects of maternal diet high in $n$-6 PUFA, $n-3$ PUFA or TFA on glucose metabolism, insulin sensitivity and fatty acid profile in male offspring. Female weanling Wistar/NIN rats were randomly assigned to receive either a diet high in linoleic acid (LA), or $\alpha$-linolenic acid (ALA), or long-chain n-3 PUFA (fish oil; FO), or TFA, for $90 \mathrm{~d}$, and mated. Upon weaning, pups were randomly divided into seven groups (mother's diet-pup's diet): LA-LA, LA-ALA, LA-FO, ALA-ALA, FO-FO, TFA-TFA and TFA-LA. At the age of 105 d, an oral glucose tolerance test, adipocyte glucose transport and muscle phospholipid fatty acid composition were measured in the pups. All animals displayed normal insulin sensitivity as evidenced by similar plasma insulin and area under the curve of insulin after an oral glucose load. Maternal intake of $n$-3 PUFA (ALA or FO) resulted in higher $n-3$ PUFA in the offspring. Plasma cholesterol and NEFA were significantly higher in the TFA-TFA group compared with the other groups. Adipocyte insulin-stimulated glucose transport and adiponectin mRNA expression were lower in TFA-TFA and TFA-LA offspring compared with the other groups. While most mother-pup fatty acid combinations did not influence the measured variables in the pups, these results indicate that maternal intake of TFA led to an unfavourable profile in the pups through to the age of $105 \mathrm{~d}$, whether the pups consumed TFA, or not.

Fetal programming: Insulin sensitivity: Rats: Dietary fatty acids

Several animal and epidemiological studies have shown that disturbances in the in utero environment may programme the fetus for the development of adult diseases in later life ${ }^{(1,2)}$. In India, the prevalence of low birth weight due to maternal malnutrition and poor antenatal care continues to be a major public health problem. Further, the metabolic syndrome, recognised as a major determinant of diet-related chronic diseases (type 2 diabetes and CHD), is widely prevalent. Insulin resistance is the underlying feature of the metabolic syndrome. Although the mechanism involved in the fetal programming of adult diseases has remained unknown, recent evidence indicates that nutritional imbalances during pregnancy cause perturbation of prenatal development which in turn permanently alters the structure, function and metabolism of tissues and organs ${ }^{(3)}$. Such alteration reduces fetal and postnatal growth resulting in increased susceptibility to dietrelated diseases in adult life.

Dietary fatty acids are known to have effects on insulin sensitivity. Changing the fatty acid content of the diet potentially influences insulin action, with SFA decreasing insulin sensitivity and PUFA increasing insulin sensitivity ${ }^{(4)}$. Furthermore, PUFA and their metabolites regulate the expression of several genes involved in glucose and lipid metabolism $^{(5)}$. Both linoleic acid (LA) and $\alpha$-linolenic acid (ALA) and their long-chain PUFA derivatives are required during normal fetal development to support the synthesis of structural lipids of brain and retinal tissue ${ }^{(6)}$. Studies have documented that an increased intake of long-chain $n-3$ PUFA during pregnancy increases the length of gestation and birth size, indicating that maternal long-chain PUFA status during pregnancy is critical for the development of the fetus ${ }^{(7)}$.

Several animal models have been established to investigate the possible role of the fetal environment in determining future susceptibility to adult diseases and the underlying mechanism. These include maternal protein restriction ${ }^{(8,9)}$, maternal energy restriction ${ }^{(10)}$ and maternal mineral restriction $^{(11,12)}$ in the rat. However, the potential role of dietary fatty acids has not been fully explored. Studies have shown that maternal intake of a high-fat diet predisposes the offspring to development of the metabolic syndrome ${ }^{(13)}$. Another study demonstrated that maternal intake of SFA predisposes the offspring to insulin resistance whereas long-chain $n$-3 PUFA had no effect ${ }^{(14)}$. Recent studies have shown that perinatal $n$-3 PUFA deficiency predisposes the offspring to hypertension in adult life ${ }^{(15)}$. Further, postnatal $n-3$ fatty acid supplementation has been shown to prevent the adverse effects of maternal glucocorticoid exposure ${ }^{(16)}$. In addition, maternal supplementation of long-chain $n$-3 PUFA to a diet containing marginal protein has been shown to be beneficial in maintaining circulating glucose, insulin, cholesterol and homocysteine levels in the adult offspring ${ }^{(17)}$. 
In recent years, the intake of n-6 PUFA and trans-fatty acids (TFA) has increased whereas $n$-3 PUFA intake has decreased. Studies on dietary fat intake among Indians showed that $n-6$ PUFA requirements are fully met owing to their high levels in cereal, millet and vegetable oils but $n-3$ PUFA intake is low and therefore the $n-6: n-3$ ratio is high $^{(18)}$. This change in dietary habits might be responsible for the increased prevalence of diet-related chronic diseases. Dietary $n-3$ PUFA has been shown to exert beneficial effects on insulin sensitivity and the development of type 2 diabetes ${ }^{(19,20)}$. A high intake of $n-6$ PUFA has been shown to have a negative impact on glucose metabolism and insulin sensitivity ${ }^{(19)}$. Further, in sucrose-induced insulin-resistant rats, we reported that substitution of dietary ALA (2.3\% energy (en\%), n-6:n-3 ratio 2) or long-chain (n-3) PUFA $(0.56$ en $\%, n-6: n-3$ ratio 10$)$ for LA increased peripheral insulin sensitivity ${ }^{(21,22)}$. We also showed that both SFA and TFA decreased insulin sensitivity and, compared with SFA, TFA decreased the insulin sensitivity to a greater extent ${ }^{(23,24)}$. TFA has been shown to inhibit PUFA biosynthesis resulting in a decreased formation of long-chain PUFA ${ }^{(25)}$. In view of increasing interest in the role of long-chain PUFA during fetal and early development and the potential adverse effects of TFA on long-chain PUFA synthesis, there has been more concern on the intake of TFA during pregnancy ${ }^{(26)}$. Although the role of dietary PUFA and TFA on insulin sensitivity in adult animals is clear, the effects during pregnancy are less explored. The aim of the present study was to investigate the effects of maternal dietary fatty acid composition on metabolic programming of the offspring. We used dietary fatty acids which reflect current intake and recommendations: a high-18:2n-6 diet (LA diet), a diet rich in 18:3n-3 (ALA diet), a diet high in long-chain $n-3$ PUFA (FO diet) and an enriched TFA diet. We measured glucose tolerance, insulin sensitivity, lipid metabolism and expression of genes associated with insulin sensitivity of the offspring of dams fed different diets.

\section{Materials and methods}

\section{Animals, diet and experimental design}

All the procedures involved in animal experiments were conducted in accordance with the guidelines of the institutional animal ethical committee. Sixteen male and thirty-two female Wistar/NIN rats, aged 3 weeks, were obtained from the National Centre for Laboratory Animal Sciences (Hyderabad, India). The animals were housed individually in polypropylene cages at $21 \pm 1^{\circ} \mathrm{C}$ with a $12 \mathrm{~h}$ light-dark cycle and fed a cereal-pulse-based diet (Table 1). In male rats groundnut oil was the source of added fat. The female rats were randomly divided into four groups; the added fat source was from various oil blends. The diet provided (by energy) $59 \%$ carbohydrates, $20 \%$ protein and $21 \%$ fat. The added fat components of the diet comprised either $45 \%$ safflower-seed oil and $55 \%$ palm oil (LA diet), $30 \%$ groundnut oil, $30 \%$ palm oil and $40 \%$ linseed oil (ALA diet), $40 \%$ groundnut oil, $50 \%$ palm oil and $10 \%$ fish oil (FO diet) or $50 \%$ safflower-seed oil and $50 \%$ partially hydrogenated vegetable oil (TFA diet). These oil blends were designed so that the fatty acids from both added oils (vegetable oils, FO and/or
Table 1. Composition of the maternal and pup $\operatorname{diet}^{\star}$

\begin{tabular}{lc}
\hline Ingredients & $\mathrm{g} / 100 \mathrm{~g}$ \\
\hline Bengal gram & 50.0 \\
Wheat & 22.5 \\
Oil & 5.0 \\
Starch & 6.0 \\
Casein & 4.0 \\
Milk powder & 7.0 \\
Salt mixture† & 4.0 \\
Vitamin mixtureł & 0.5 \\
Cellulose & 1.0 \\
Total energy $(\mathrm{kJ} / 100 \mathrm{~g})$ & 1561 \\
\hline
\end{tabular}

*All the experimental diets (linoleic acid diet, $\alpha$-linolenic acid diet, fish oil diet and trans-fatty acid diet) were identical except for the type of fat.

†Salt mixture contained $(\mathrm{g} / 100 \mathrm{~g}): \mathrm{CaCO}_{3}, 3.81 ; \mathrm{KH}_{2} \mathrm{PO}_{4}, 3.59 ; \mathrm{NaCl}, 13.93$; $\mathrm{MgSO}_{4} .7 \mathrm{H}_{2} \mathrm{O}, 5.73 ; \mathrm{MnSO}_{4} . \mathrm{H}_{2} \mathrm{O}, 0.4 ; \mathrm{KI}, 0.8 ; \mathrm{ZnSO}_{4}, 0.48 ; \mathrm{CuSO}_{4} .5 \mathrm{H}_{2} \mathrm{O}$, $0.048 ; \mathrm{CoCl}_{2} \cdot 6 \mathrm{H}_{2} \mathrm{O}, 0.002 ; \mathrm{FeSO}_{4} .7 \mathrm{H}_{2} \mathrm{O}, 2.2 ; \mathrm{Na}_{2} \mathrm{SeO}_{3}, 0.001 ;\left(\mathrm{NH}_{4}\right)_{6} \mathrm{MO}_{7} \mathrm{O}_{24} \cdot 4$ $\mathrm{H}_{2} \mathrm{O}, 0.0008$; made up to $100 \mathrm{~g}$ with starch.

$\ddagger$ Vitamin mixture contained $(\mathrm{g} / 100 \mathrm{~g})$ : inositol, 2 ; $p$-amino benzoic acid, 2; thiamin, 0.05; riboflavin, 0.08; niacin, 0.2; menadione, 0.03; pyridoxine, 0.12; folic acid, 0.02 ; calcium pantothenate, 0.24 ; cyanacobalamin, 0.0003 ; biotin, 0.008 ; vitamin $\mathrm{E}$ acetate, 1.2; made up to $100 \mathrm{~g}$ with starch.

partially hydrogenated vegetable oil) and fat from components other than added fats (endogenous fat) together furnished different ratios of $18: 2 n-6: 18: 3 n-3$ (LA group about 28 and ALA group about 2.5), $18: 2 n-6: 18: 3 n-3+$ long-chain $n-3$ PUFA (FO group about 10) or $18: 2 n-6: 18: 3 n-3$ (TFA group 28 plus TFA). The fatty acid compositions of the diets (Table 2) were determined by GC as described earlier ${ }^{(27)}$. Diets were prepared once weekly by adding the oils to the base mixture containing other nutrients (Table 1) and stored at $4^{\circ} \mathrm{C}$ before use. The oils and base mixtures were stored at $4^{\circ} \mathrm{C}$ until preparation of the diet. FO was kept under an atmosphere of $\mathrm{N}_{2}$ and stored at $-20^{\circ} \mathrm{C}$. All the diets were supplemented with $\alpha$-tocopherol $(0.015 \mathrm{~g} / \mathrm{kg}$ diet $)$ to prevent oxidation. Rats were given fresh diet every day and had free access to food and water. The daily food intake and weekly body weight of individual rats were recorded.

After $90 \mathrm{~d}$ feeding with the above diets, blood was collected from the retro-orbital sinus in EDTA tubes after overnight fasting and plasma was separated and stored at $-70^{\circ} \mathrm{C}$ for the analysis of glucose, insulin, TAG and cholesterol. After $5 \mathrm{~d}$ of bleeding, the rats were mated with males (two females + one male) and the pregnant rats continued to consume their respective diets throughout gestation. After delivery, the litter weight and size for each mother were recorded. The litter size of each mother was equalised to eight and the mother was

Table 2. Fatty acid composition of diets (added fat + from other dietary ingredients)

\begin{tabular}{|c|c|c|c|c|}
\hline Fatty acids (g/100 g diet) & LA diet & ALA diet & FO diet & TFA diet \\
\hline$\Sigma$ SFA & 1.88 & 1.65 & 1.94 & 1.82 \\
\hline$\Sigma$ MUFA & $2 \cdot 17$ & $2 \cdot 27$ & 2.09 & $2 \cdot 26$ \\
\hline Cis & $2 \cdot 17$ & $2 \cdot 27$ & 2.09 & 1.80 \\
\hline Trans & 0.00 & 0.00 & 0.00 & 0.46 \\
\hline$\Sigma$ PUFA & $4 \cdot 14$ & $4 \cdot 21$ & $4 \cdot 14$ & $4 \cdot 10$ \\
\hline $18: 2 n-6$ & 4.00 & 3.02 & 3.79 & 3.95 \\
\hline $18: 3 n-3$ & 0.14 & $1 \cdot 19$ & 0.15 & 0.14 \\
\hline Long-chain n-3 PUFA & 0.00 & 0.00 & 0.20 & 0.00 \\
\hline$n-6: n-3$ & 28 & $2 \cdot 50$ & 11 & 28 \\
\hline
\end{tabular}

LA, linoleic acid; ALA, $\alpha$-linolenic acid; FO, fish oil; TFA, trans-fatty acid. 
continued on the respective experimental diets. At weaning (at age $21 \mathrm{~d}$ ), only male pups born to LA-group mothers were randomly split into three groups. One group was continued on the LA diet (LA-LA); the other two groups were switched to the ALA diet (LA-ALA) or the FO diet (LA-FO). Pups born to the ALA group (ALA-ALA) and the FO group (FO-FO) were continued on the respective mother's diet. Pups born to TFA mothers were split into two groups. One group was continued on the TFA diet (TFA-TFA) and the other group (TFA-LA) was switched on to the LA diet. Since female sex hormones may have a protective role against the development of insulin resistance, only male pups were studied ${ }^{(28,29)}$. Fig. 1 gives the schematic representation of the feeding protocol used.

\section{Biochemical methods}

The oral glucose tolerance test was done at the end of $90 \mathrm{~d}$ feeding of the experimental diets to the pups. Pups were given D-glucose ( $3 \mathrm{~g}$ glucose/kg body weight) through gastric administration after being deprived of food for $18 \mathrm{~h}$. Blood was collected from the retro-orbital sinus in EDTA tubes at 0, 30, 60 and $120 \mathrm{~min}$. Plasma was separated and stored at $-70^{\circ} \mathrm{C}$ until analysis. Areas under the curve for glucose and insulin were calculated by the trapezoidal rule. Fasting plasma glucose (glucose oxidase/peroxidase kit), TAG (glycerol phosphate oxidase kit) and total cholesterol (cholesterol oxidase-peroxidase kit) were measured using enzymic kits from Biosystems (Barcelona, Spain). Plasma NEFA were measured using an acyl CoA synthetase-oxidase-peroxidase kit from Roche (Basel, Switzerland) and plasma insulin by a RIA kit from BRIT (Board of Radiation \& Isotope Technology, Mumbai, India).

At the end of the experimental period $(105 \mathrm{~d})$, the animals were killed by $\mathrm{CO}_{2}$ asphyxiation. Epididymal and retroperitoneal fat pads and diaphragm were dissected, weighed and snap-frozen in liquid $\mathrm{N}_{2}$ and stored at $-70^{\circ} \mathrm{C}$. A part of epididymal adipose tissue was used for isolation of adipocytes by the collagenase digestion method ${ }^{(21)}$.

Adipocyte lipolysis was assayed by measuring glycerol release into the incubation medium ${ }^{(21,22)}$. Adipocytes were incubated at $37^{\circ} \mathrm{C}$ for $2 \mathrm{~h}$ in Krebs-Ringer-HEPES (KRH) buffer $(\mathrm{pH} 7.4)$ in a final volume of $0.5 \mathrm{ml}$. Basal lipolysis was measured without the addition of hormone. Hormonestimulated lipolysis was assessed by adding various concentrations of noradrenaline. The antilipolytic effect of insulin was assessed by incubating adipocytes with $1 \mu \mathrm{M}$-noradrenaline (maximum stimulating concentration) and various concentrations of insulin. After $2 \mathrm{~h}$ of incubation the cells were separated from the medium by brief centrifugation. The infranatant fraction below the cell layer was removed and glycerol content was estimated.

Basal and insulin-stimulated glucose transport was measured as described ${ }^{(21,22)}$. Adipocytes were pre-incubated with and without various concentrations of porcine insulin at $37^{\circ} \mathrm{C}$ for $45 \mathrm{~min}$. The cells were incubated with $2-\left[1,2-{ }^{3} \mathrm{H}\right]$ deoxyglucose at a concentration of $0 \cdot 1 \mathrm{~mm}$ in $\mathrm{KRH}$ buffer $(\mathrm{pH} 7 \cdot 4)$. The assay was terminated at the end of 3 min by transferring the assay mixture to a microcentrifuge tube containing silicone oil. The tube was centrifuged at $4000 \mathrm{rpm}$ for $3 \mathrm{~min}$. The top layer containing adipocytes was transferred to a liquid scintillation vial and radioactivity associated with the adipocytes was measured in a liquid scintillation counter. All data were corrected for non-specific transport by measuring glucose transport in the presence of $0.3 \mathrm{~mm}$-phloretin.

Diaphragm total lipids were extracted using the method of Folch $^{(30)}$. Neutral lipids were separated from phospholipids by TLC on silica gel $\mathrm{G}$ with hexane-diethyl ether-acetic acid (80:20:1, by vol.) and the fatty acid methyl esters

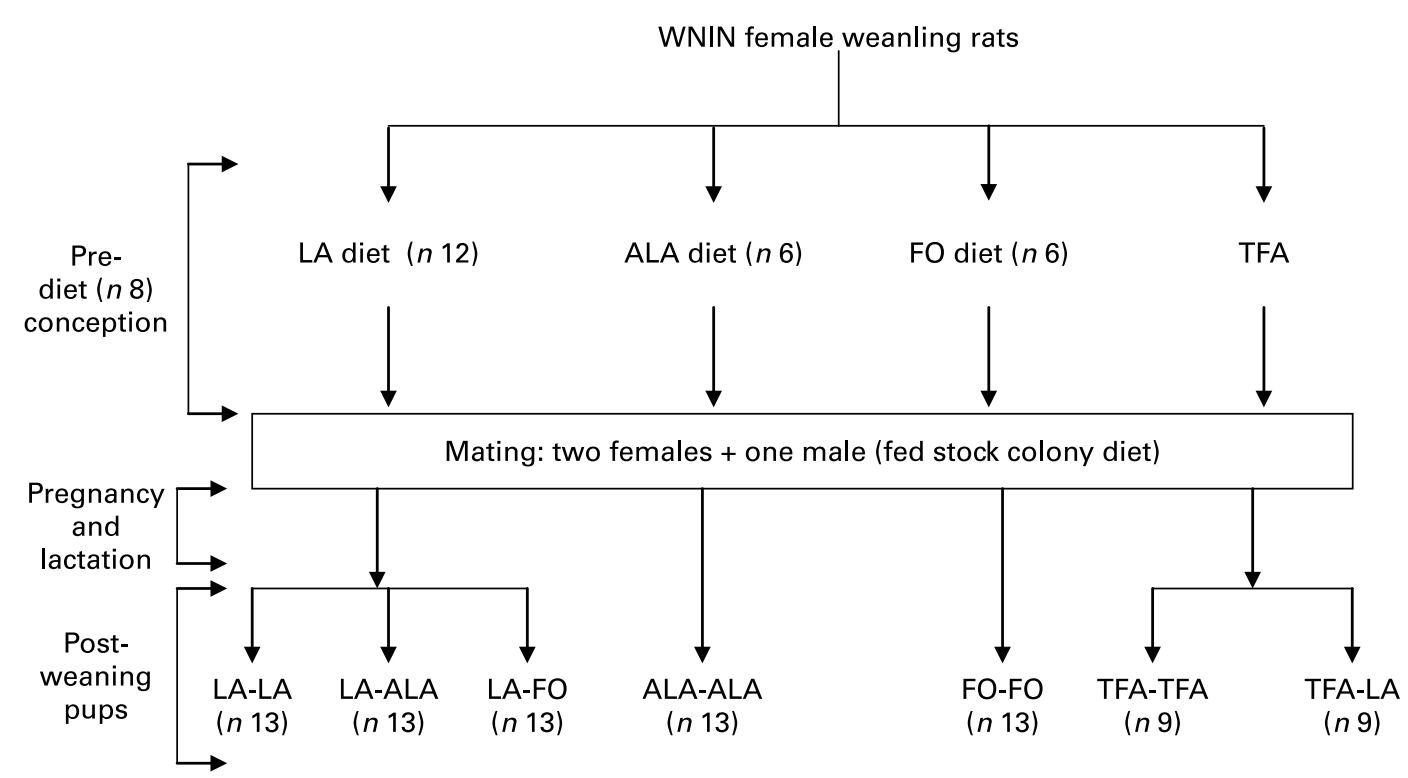

Fig. 1. Feeding protocol of different groups of mothers and offspring. WNIN, Wistar/NIN; LA-LA, mothers received the linoleic acid (LA) diet and pups were continued on the same diet from weaning; LA-ALA, mothers received the LA diet and pups were fed with the $\alpha$-linolenic acid (ALA) diet from weaning; LA-FO, mothers received the LA diet and pups were fed with the fish oil (FO; long-chain $n$-3 PUFA) diet; ALA-ALA, mothers received the ALA diet and pups were continued on the same diet; FO-FO, mothers received the long-chain $n$-3 PUFA diet and pups were continued on the same diet; TFA-TFA, mothers received the trans-fatty acid (TFA) diet and pups continued on the same diet; TFA-LA, mothers received the TFA diet and pups were fed with the LA diet from weaning. 
were analysed by GC using an SP-2330 capillary column $(30 \mathrm{~m} \times 0.25 \mathrm{~mm}$; Supelco, Bellefonte, PA, USA) as described earlier ${ }^{(27)}$.

\section{RNA extraction and reverse transcriptase polymerase chain reaction}

Total RNA was isolated from epididymal adipose tissue using TRI reagent (Sigma, St Louis, MO, USA) according to the manufacturer's instructions. Adipose tissue from two rats in each group was pooled for the extraction of RNA. RT-PCR was performed using a single tube access RT-PCR kit (Promega, Madison, WI, USA). The method couples reverse transcription (RT) of total RNA with PCR amplification using gene-specific primers, in the presence of avian myeloblastosis virus (AMV) RT and Thermus filiformis (Tfl) DNA polymerase respectively. Primer sequences were (forward and reverse): rat adiponectin, $5^{\prime}$-aggatccatgctactgttgcaagcgctc- $3^{\prime}$ and $5^{\prime}$-gaagcttgttggtatcatggtagagaagg- $3^{\prime}$; rat lipoprotein lipase, $5^{\prime}$-gtatcgggcccagcaacattatcc- $3^{\prime}$ and $5^{\prime}$-gccttgctggggtttcttcattc-3'; rat GLUT-4, 5'-tctcggtgctcttagtag- $3^{\prime}$ and $5^{\prime}$-ccaatctcaaagaaggccacaaa- $3^{\prime}$; rat sterol regulatory binding protein-1c, $5^{\prime}$-cgctaccgttcctctatcaatg- $3^{\prime}$ and $5^{\prime}$-cctcctccactgccacaag- $3^{\prime}$; rat TNF- $\alpha, 5^{\prime}$-ctcagcctcttctcattc- $3^{\prime}$ and $5^{\prime}$-gaacctgggagtagataag- $3^{\prime}$; rat $\beta$-actin (internal control), $5^{\prime}$ gtgggecgctctaggcacca- $3^{\prime}$ and $5^{\prime}$-cggttggcettagggttcaggggg- $3^{\prime}$. The thermal cycler conditions for PCR (thirty cycles) were as follows: initial RT for $10 \mathrm{~min}$ at $25^{\circ} \mathrm{C}$ followed by stringent RT at $48^{\circ} \mathrm{C}$ for $45 \mathrm{~min}$; inactivation of RT at $95^{\circ} \mathrm{C}$ for $5 \mathrm{~min}$; denaturation at $94^{\circ} \mathrm{C}$ for $30 \mathrm{~s}$; annealing at $55-58^{\circ} \mathrm{C}$ for $30 \mathrm{~s}$; extension at $68^{\circ} \mathrm{C}$ for $30 \mathrm{~s}$ followed by a final extension at $68^{\circ} \mathrm{C}$ for $7 \mathrm{~min}$. PCR-amplified DNA was electrophoresed on agarose gel $(1.5 \%)$ and ethidium bromide-stained bands were analysed densitometrically using the Quantity One software program (version 4.4.0; Bio-Rad, Hercules, CA, USA). Each RT-PCR experiment of individual genes was repeated at least three times. The relative mRNA expression of individual genes was calculated according to the $\beta$-actin levels (internal control). The percentage relative expression of candidate genes in different experimental groups was calculated by considering gene expression observed in the LA-LA group as 100 .

\section{Statistical analysis}

Statistical analysis was done using the SPSS statistical program (SPSS, Inc, Chicago, IL, USA). All of the values are presented as mean values with their standard errors. Data were evaluated by one-way ANOVA followed by least square difference (LSD) post hoc tests. Differences of $P<0.05$ were considered significant.

\section{Results}

Food intake, body weight, plasma lipids, glucose and insulin

Body weight, food intake, plasma glucose, insulin and lipids of the dams were similar between the groups before and after pregnancy and lactation (data not shown). The litter weight and size were also similar in all groups (data not shown). At weaning and in the post-weaning period up to

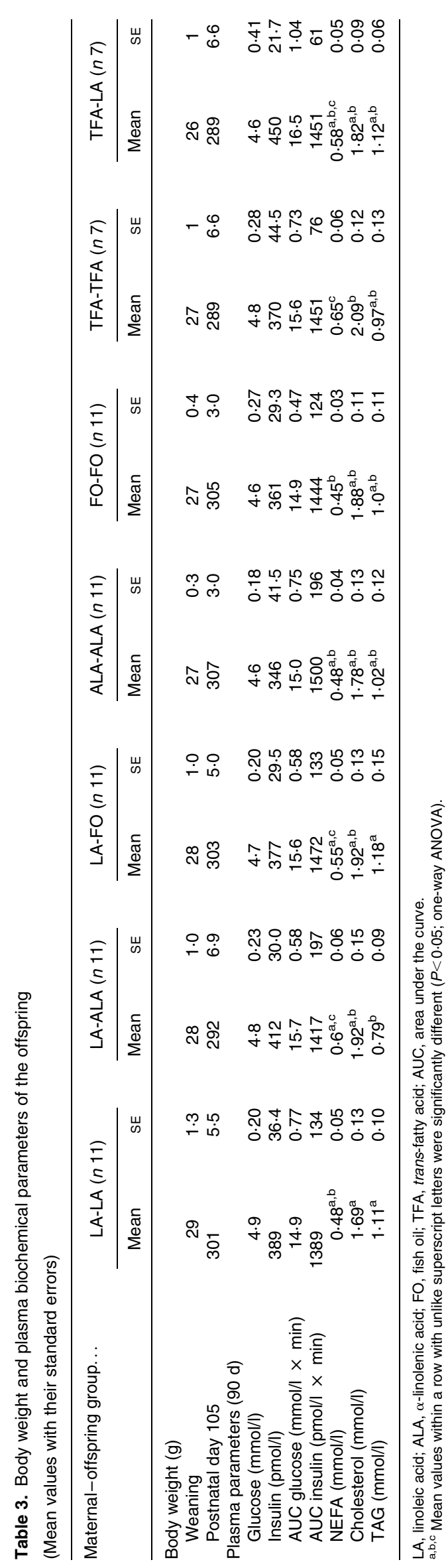


$105 \mathrm{~d}$, the body weight of the offspring in all the groups was not significantly different (Table 3). At 90 d, plasma glucose, insulin, area under the curve of glucose and area under the curve of insulin after the oral glucose load were not significantly different between the groups. Plasma TAG levels were significantly lower in the LA-ALA group compared with the other groups. The TFA-TFA group had higher plasma cholesterol and NEFA levels compared with the LALA group. Plasma levels of cholesterol and NEFA tended to be higher even after switching over the offspring to the LA diet after weaning (TFA-LA).

\section{Adipocyte lipolysis, antilipolysis and glucose transport}

Adipocyte lipolysis (basal and noradrenaline stimulated) and antilipolytic effects of insulin were similar in all groups (data not shown). Maternal intake of TFA (TFA-TFA) decreased the adipocyte insulin-stimulated glucose transport of the offspring at all the concentrations of insulin and the decrease was significant only at higher concentration. These changes were not corrected even after switching over to the LA diet devoid of TFA (TFA-LA) after weaning (Fig. 2).

\section{Diaphragm fatty acid composition}

The data on diaphragm phospholipids fatty acid composition showed no changes in SFA between the groups (Table 4). Maternal or post-weaning intake of ALA or long-chain $n-3$ PUFA altered the fatty acid composition of the offspring. As expected, the LA-ALA, LA-FO, ALA-ALA and FO-FO groups had higher $n-3$ PUFA proportions in the diaphragm phospholipids. The $n-3$ PUFA supplementation (ALA or FO) significantly decreased the levels of long-chain n-6 PUFA with an increase in the levels of long-chain n-3 PUFA (22:6n-3). Maternal intake of TFA resulted in incorporation of $18: 1$ trans.

\section{Gene expression studies}

Maternal intake of $n$-3 PUFA (ALA or long-chain $n$-3 PUFA) did not alter the mRNA expression of genes associated with insulin sensitivity in the offspring (Fig. 3). Maternal intake of dietary TFA (TFA-TFA) decreased the level of adiponectin mRNA expression (about $35 \%$ ) as compared with the LA-LA group. Switching over to the LA diet devoid of TFA from weaning (TFA-LA) did not normalise the TFA-induced decrease in adiponectin mRNA expression (Table 5).

\section{Discussion}

PUFA, particularly $n-3$ PUFA, have been shown to increase insulin sensitivity in adult animals. Our earlier studies in male rats have shown that substitution of starch with sucrose in casein-based semi-synthetic diets providing 22 en\% fat and 0.03 en $\%$ ALA induced insulin resistance by decreasing peripheral insulin sensitivity. However, supplementation of either ALA or long-chain n-3 PUFA prevented the sucroseinduced decrease in peripheral insulin sensitivity ${ }^{(21,22)}$. The maternal nutrient composition has been shown to have an impact on the growth and development of the offspring ${ }^{(3)}$. The potential role of PUFA and TFA has not been fully explored. Maternal supplementation of $n-3$ PUFA has been shown to decrease growth rate, adipose tissue mass and serum leptin levels in offspring ${ }^{(31)}$. Moreover, maternal intake of $n-6$ or $n-3$ PUFA has been shown to alter the insulin sensitivity and blood pressure in 186-d-old male offspring ${ }^{(32)}$. In the present study, maternal supplementation of PUFA ( $n-6$ or $n$-3) did not alter the glucose and lipid metabolism of the

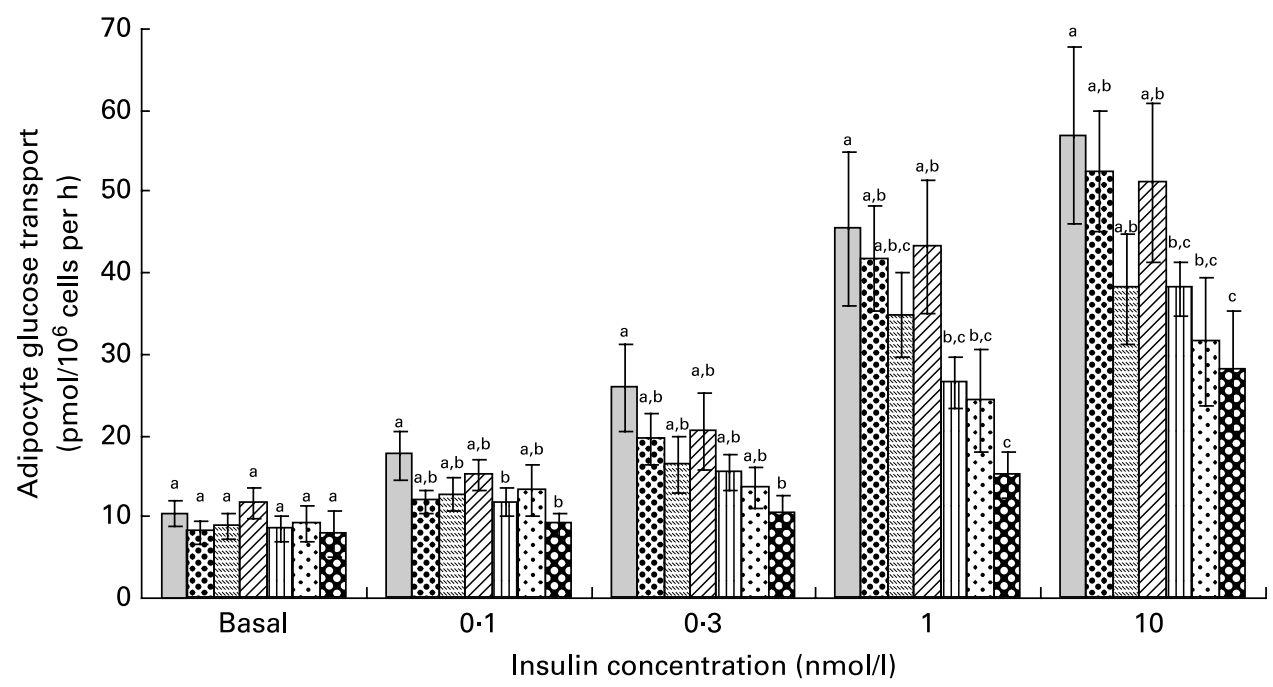

Fig. 2. Insulin-stimulated glucose uptake in isolated epididymal adipocytes from rats fed different experimental diets: ( $\square$ ), LA-LA, mothers received the linoleic acid (LA) diet and pups were continued on the same diet from weaning; ( 0 ), LA-ALA, mothers received the LA diet and pups were fed with the $\alpha$-linolenic acid (ALA) diet from weaning; ( $⿴ 囗 ⿱ 一 一)$ ), LA-FO, mothers received the LA diet and pups were fed with the fish oil (FO; long-chain $n$-3 PUFA) diet; ( $\square$ ), ALA-ALA, mothers received the ALA diet and pups were continued on the same diet; (四), FO-FO, mothers received the long-chain $n$-3 PUFA diet and pups were continued on the same diet; ( $\nabla)$, TFA-TFA, mothers received the trans-fatty acid (TFA) diet and pups continued on the same diet; ( $\mathbf{Q}$ ), TFA-LA, mothers received the TFA diet and pups were fed with the LA diet from weaning. Adipocytes were pre-incubated at $37^{\circ} \mathrm{C}$ for $45 \mathrm{~min}$ in the absence and presence of insulin at the concentrations indicated. Glucose uptake was measured as described in Materials and methods. Values are means $(n 7)$, with standard errors represented by vertical bars. ${ }^{a, b, c}$ At a particular concentration of insulin, mean values with unlike letters were significantly different $(P<0.05$; one-way ANOVA). 
|

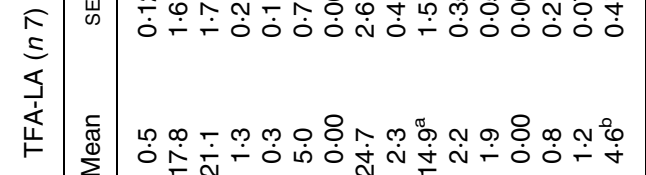

山

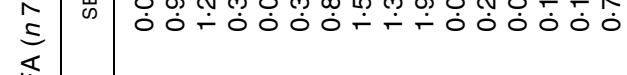

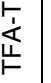

น

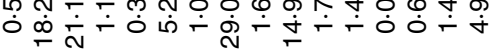

馬|

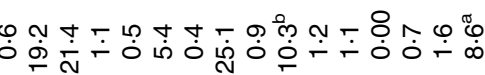

更|

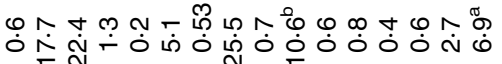
พับ ๓ำ n का

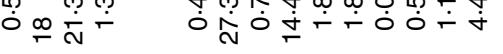

male offspring up to age $105 \mathrm{~d}$ of postnatal life. Several factors such as age and sex have been shown to have major effect on metabolic programming. The lack of effect of PUFA on metabolic programming in the present study could be due to the short duration of the study. Further, the effects in females need to be studied.

Several studies have documented that dietary ALA increases long-chain $n$-3 PUFA in various tissues in rats $^{(33,34)}$. Further, prenatal deficiency of ALA has been shown to decrease $22: 6 n-3$ proportions in the offspring even after repletion of the diet with ALA after weaning ${ }^{(35)}$. Down-regulation of $\Delta 6$-desaturase has been suggested to be the cause of low levels of $22: 6 n-3$. The present study has demonstrated that increasing ALA or long-chain $n$-3 PUFA elicits beneficial effects with respect to $n-3$ PUFA status as evidenced by an increase in long-chain $n-3$ PUFA levels in diaphragm membrane phospholipids.

In recent years, due to changes in dietary patterns, the intake of TFA has increased. TFA are transferred from mother to fetus ${ }^{(36)}$. Both the fetus and the breast-fed baby are consequently exposed to TFA corresponding to mother's intake. Animal experiments suggest that a high intake of TFA inhibits the formation of long-chain PUFA which are important in fetal and infant growth and in visual and central nervous system development ${ }^{(37)}$. Recent studies have shown that the mother's intake of TFA is negatively associated with levels of long-chain PUFA in the blood of newborn infant and therefore have suggested the minimisation of intake of TFA during pregnancy ${ }^{(38)}$. A high intake of TFA has been shown to decrease long-chain $n-3$ PUFA levels in the plasma and liver of pregnant and fetal rats ${ }^{(39)}$. Epidemiological studies show that the risk of the development of type 2 diabetes is associated with TFA intake ${ }^{(40)}$. More recent longterm studies in monkeys also showed that dietary TFA increases adiposity and decreases insulin sensitivity ${ }^{(41)}$. Our earlier study showed that a casein-based semi-synthetic diet providing $10 \%$ fat and 3 en $\%$ TFA increased plasma TAG and decreased insulin sensitivity in male rats ${ }^{(23,24)}$. In the present study, maternal dietary TFA (1 en\%) had no effect on plasma glucose, insulin levels or oral glucose tolerance (glucose and insulin areas under the curve) in the offspring until the age of $105 \mathrm{~d}$. In addition, there were no changes in the levels of long-chain PUFA in diaphragm phospholipids. Altered adiposity and lipid metabolism have been suggested to be early changes before the animals become insulin resistant $^{(42)}$. In the present study, maternal dietary TFA altered cholesterol and lipid metabolism as evidenced by increases in plasma cholesterol and NEFA in the offspring; these changes were not reversed even after switching over to the TFA-free diet from weaning. Recent studies indicate that adipose tissue secretes a number of proteins called adipocytokines which play a major role in whole-body insulin sensitivity ${ }^{(43)}$. Leptin and adiponectin increase insulin sensitivity whereas TNF- $\alpha$ and resistin decrease insulin sensitivity. Dietary SFA and TFA have been shown to differentially alter the adipose tissue expression of genes associated with insulin sensitivity in rats ${ }^{(44)}$. In the present study we also measured the expression levels of some of the genes associated with glucose and lipid metabolism in adipose tissue and also insulin-stimulated glucose transport. Interestingly, maternal dietary TFA down-regulated the expression of adiponectin and 


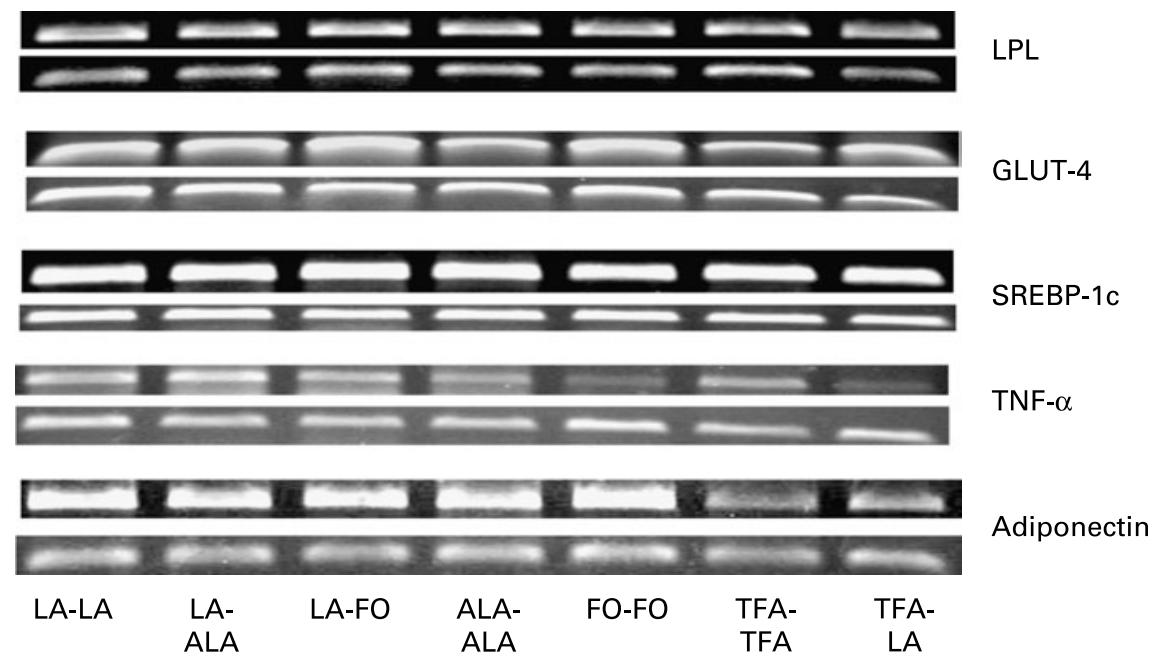

Fig. 3. Effect of dietary fatty acids on adipose tissue gene expression in the offspring. Total RNA was extracted from epididymal tissue of rats fed different experimental diets: LA-LA, mothers received the linoleic acid (LA) diet and pups were continued on the same diet from weaning; LA-ALA, mothers received the LA diet and pups were fed with the $\alpha$-linolenic acid (ALA) diet from weaning; LA-FO, mothers received the LA diet and pups were fed with the fish oil (FO; long-chain $n$ - 3 PUFA) diet; ALA-ALA, mothers received the ALA diet and pups were continued on the same diet; FO-FO, mothers received the long-chain $n$-3 PUFA diet and pups were continued on the same diet; TFA-TFA, mothers received the trans-fatty acid (TFA) diet and pups continued on the same diet; TFA-LA, mothers received the TFA diet and pups were fed with the LA diet from weaning. mRNA levels of lipoprotein lipase (LPL), GLUT-4, sterol regulatory binding protein (SREBP-1c), TNF- $\alpha$ and adiponectin were analysed using RT-PCR. Relative mRNA expression was measured in relation to $\beta$-actin mRNA levels. Agarose gel electrophoresis (1.5\%) of RT-PCR products (upper lane) and corresponding $\beta$-actin gene (lower lane).

Table 5. Adipose tissue gene expression of the offspring fed with different experimental diets* (Mean values with their standard errors of three independent experiments)

\begin{tabular}{|c|c|c|c|c|c|c|c|c|c|c|}
\hline \multirow[b]{2}{*}{ Diet groups } & \multicolumn{2}{|c|}{ Lpl } & \multicolumn{2}{|c|}{ GLUT-4 } & \multicolumn{2}{|c|}{ SREBP-1C } & \multicolumn{2}{|c|}{ TNF- $\alpha$} & \multicolumn{2}{|c|}{ Adiponectin } \\
\hline & Mean & SE & Mean & SE & Mean & SE & Mean & SE & Mean & SE \\
\hline LA-LA & $100 \cdot 00$ & & $100 \cdot 00$ & & $100 \cdot 00$ & & $100 \cdot 00$ & & 100.00 & \\
\hline LA-ALA & $107 \cdot 34$ & 6.48 & 104.77 & 7.04 & 108.44 & 1.39 & $108 \cdot 04$ & $6 \cdot 61$ & 91.88 & $6 \cdot 22$ \\
\hline LA-FO & 109.90 & $5 \cdot 14$ & 110.41 & 5.49 & 111.67 & $3 \cdot 21$ & 105.06 & 7.05 & 97.99 & 3.29 \\
\hline ALA-ALA & $102 \cdot 64$ & $7 \cdot 20$ & 95.89 & 5.06 & 107.96 & $8 \cdot 11$ & 97.58 & $7 \cdot 37$ & 93.30 & 4.97 \\
\hline FO-FO & 94.02 & 1.35 & 92.30 & $5 \cdot 37$ & 91.29 & $5 \cdot 79$ & 91.02 & $6 \cdot 26$ & 99.72 & 1.00 \\
\hline TFA-TFA & 101.52 & 9.06 & 95.67 & $2 \cdot 32$ & $117 \cdot 31$ & 8.43 & $112 \cdot 13$ & 2.04 & 65.11 & 4.29 \\
\hline TFA-LA & $114 \cdot 22$ & $6 \cdot 88$ & 103.39 & $4 \cdot 70$ & 103.48 & $10 \cdot 03$ & 91.02 & $8 \cdot 16$ & $66 \cdot 75$ & 3.93 \\
\hline
\end{tabular}

LPL, lipoprotein lipase; sterol regulatory binding protein; LA, linoleic acid; ALA, $\alpha$-linolenic acid; FO, fish oil; TFA, trans-fatty acid.

* Relative mRNA expression was measured in relation to $\beta$-actin mRNA levels. Percentage relative expression of all mRNA was calculated considering the relative mRNA expression observed in the LA-LA group as 100 .

decreased insulin-stimulated glucose transport in the offspring; these changes were not reversible even if the offspring were switched over to the TFA-free diet from weaning. These observations suggested the programming effect of the TFA diet. In line with these results, a recent study showed that intra-uterine/prenatal exposure of dietary TFA programmed the hypothalamus feeding control mechanism ${ }^{(45)}$. Despite alterations in glucose transport, the expression of adipocyte GLUT-4 was not altered in the present study. Membrane lipid composition and fluidity has been shown to play a major role in glucose transport ${ }^{(46,47)}$. Although we did not measure the adipocyte membrane fluidity, the decrease in glucose transport may be due to changes in membrane fluidity and defective translocation of GLUT-4 to the adipocyte plasma membrane.

In conclusion, the results of the present study indicate that maternal diets high in $n$-6 PUFA (about 10 en\%) or $n-3$
PUFA (ALA about 3 en $\%$, or about 0.5 en $\%$ long-chain $n$-3 PUFA) did not alter insulin sensitivity of the male offspring at postnatal day 105. However, maternal intake of TFA even at low levels (about 1 en\%) evoked programming within the metabolic process of the offspring which may predispose the offspring to the development of insulin resistance in adult life. These findings strengthen the importance of restricting the intake of TFA during pregnancy and lactation.

\section{Acknowledgements}

Financial support for the present study was from the National Institute of Nutrition, Hyderabad. India.

A. I. performed the data analysis and drafting of the manuscript; Ghafoorunissa conceived of the study, designed the study, implemented the study, analysed the data and critically 
revised the manuscript; S. B. performed the gene expression study and drafting of the relevant section of the manuscript; N. Z. E. critically revised the gene expression study section.

The authors thank K. Swarupa Rani, R. Laxmi, A. Vani and A. Brinda for their technical assistance in the care and maintenance of the animals, sample collection and biochemical estimations.

The authors disclose that they have no financial interest or commercial sponsor for the present study.

\section{References}

1. Barker DJ (2002) Fetal programming of coronary heart disease. Trends Endocrinol Metab 13, 364-368.

2. Simmons R (2005) Developmental origins of adult metabolic disease: concepts and controversies. Trends Endocrinol Metab 16, 390-394.

3. Holness MJ, Langdown ML \& Sugden MC (2000) Early-life programming of susceptibility to dysregulation of glucose metabolism and the development of type 2 diabetes mellitus Biochem J 349, 657-665.

4. Lichtenstein AH \& Schwab US (2000) Relationship of dietary fat to glucose metabolism. Atherosclerosis 150, 227-243.

5. Clarke SD (2001) Polyunsaturated fatty acid regulation of gene transcription: a molecular mechanism to improve the metabolic syndrome. J Nutr 131, 1129-1132.

6. Lauritzen L, Hansen HS, Jørgensen MH, et al. (2001) The essentiality of long chain $n-3$ fatty acids in relation to development and function of the brain and retina. Prog Lipid Res $\mathbf{4 0}$, $1-94$.

7. Olsen SF, Sørensen JD, Secher NJ, et al. (1992) Randomised controlled trial of effect of fish-oil supplementation on pregnancy duration. Lancet 339, 1003-1007.

8. Ozanne SE, Wang CL, Coleman N, et al. (1996) Altered muscle insulin sensitivity in the male offspring of proteinmalnourished rats. Am J Physiol Endocrinol Metab 271, E1128-E1134.

9. Fernandez-Twinn DS, Wayman A, Ekizoglou S, et al. (2005) Maternal protein restriction leads to hyperinsulinemia and reduced insulin-signaling protein expression in 21-mo-old female rat offspring. Am J Physiol Regul Integr Comp Physiol 288, R368-R373.

10. Kind KL, Clifton PM, Grant PA, et al. (2003) Effect of maternal feed restriction during pregnancy on glucose tolerance in the adult guinea pig. Am J Physiol Regul Integr Comp Physiol 284, R140-R152.

11. Lewis RM, Petry CJ, Ozanne SE, et al. (2001) Effects of maternal iron restriction in the rat on blood pressure, glucose tolerance, and serum lipids in the 3-month-old offspring. Metabolism 50, 562-567.

12. Venu L, Kishore YD \& Raghunath M (2005) Maternal and perinatal magnesium restriction predisposes rat pups to insulin resistance and glucose intolerance. J Nutr $\mathbf{1 3 5}$, 1353-1358.

13. Buckley AJ, Keserü B, Briody J, et al. (2005) Altered body composition and metabolism in the male offspring of high fatfed rats. Metabolism 54, 500-507.

14. Siemelink M, Verhoef A, Dormans JA, et al. (2002) Dietary fatty acid composition during pregnancy and lactation in the rat programs growth and glucose metabolism in the offspring. Diabetologia 45, 1397-1403.

15. Armitage JA, Pearce AD, Sinclair AJ, et al. (2003) Increased blood pressure later in life may be associated with perinatal n-3 fatty acid deficiency. Lipids 38, 459-464.
16. Wyrwoll CS, Mark PJ, Mori TA, et al. (2006) Prevention of programmed hyperleptinemia and hypertension by postnatal dietary omega-3 fatty acids. Endocrinology 147, 599-606.

17. Joshi S, Rao S, Golwilkar A, et al. (2003) Fish oil supplementation of rats during pregnancy reduces adult disease risks in their offspring. J Nutr 133, 3170-3174.

18. Ghafoorunissa (1996) Fats in Indian diets and their nutritional and health implications. Lipids 31, S287-S291.

19. Storlien LH, Jenkins AB, Chisholm DJ, et al. (1991) Influence of dietary fat composition on development of insulin resistance in rats. Relationship to muscle triglyceride and omega-3 fatty acids in muscle phospholipid. Diabetes 40, 280-289.

20. Nettleton JA \& Katz R (2005) n-3 Long-chain polyunsaturated fatty acids in type 2 diabetes: a review. $J$ Am Diet Assoc 105, $428-440$.

21. Ghafoorunissa, Ibrahim A \& Natarajan S (2005) Substituting dietary linoleic acid with $\alpha$-linolenic acid improves insulin sensitivity in sucrose fed rats. Biochim Biophys Acta 1733, 67-75.

22. Ghafoorunissa, Ibrahim A, Rajkumar L, et al. (2005) Dietary (n-3) long chain polyunsaturated fatty acids prevent sucrose induced insulin resistance in rats. J Nutr 135, 2634-2638.

23. Natarajan S, Ibrahim A \& Ghafoorunissa (2005) Dietary trans fatty acids alter diaphragm phospholipid fatty acid composition, triacylglycerol content and glucose transport in rats. Br J Nutr 93, 829-833.

24. Ibrahim A, Natrajan S \& Ghafoorunissa R (2005) Dietary transfatty acids alter adipocyte plasma membrane fatty acid composition and insulin sensitivity in rats. Metabolism 54, 240-246.

25. Larqué E, García-Ruiz PA, Perez-Llamas F, et al. (2003) Dietary trans fatty acids alter the compositions of microsomes and mitochondria and the activities of microsome $\Delta 6$-fatty acid desaturase and glucose-6-phosphatase in livers of pregnant rats. J Nutr 133, 2526-2531.

26. Innis SM (2006) Trans fatty intakes during pregnancy, infancy and early childhood. Atheroscler Suppl 7, 17-20.

27. Ghafoorunissa, Reddy V \& Sesikaran B (1995) Palmolein and groundnut oil have comparable effects on blood lipids and platelet aggregation in healthy Indian subjects. Lipids $\mathbf{3 0}$, $1163-1169$

28. Sugden MC \& Holness MJ (2002) Gender specific programming of insulin secretion and action. $J$ Endocrinol 175, 757-767.

29. Galipeau D, Verma S \& McNeill JH (2002) Female rats are protected against fructose induced changes in metabolism and blood pressure. Am J Physiol Heart Circ Physiol 283, $\mathrm{H} 2478-\mathrm{H} 2484$.

30. Folch J, Lees M \& Sloane Stanley GH (1957) A simple method for the isolation and purification of total lipides from animal tissues. J Biol Chem 226, 497-509.

31. Korotkova M, Gabrielsson B, Lönn M, et al. (2002) Leptin levels in rat offspring are modified by the ratio of linoleic to $\alpha$-linolenic acid in the maternal diet. $J$ Lipid Res 43, $1743-1749$.

32. Korotkova M, Gabrielsson BG, Holmäng A, et al. (2005) Gender-related long-term effects in adult rats by perinatal dietary ratio of $n-6 / n-3$ fatty acids. Am J Physiol Regul Integr Comp Physiol 288, R575-R579.

33. Kim HK \& Choi H (2001) Dietary $\alpha$-linolenic acid lowers postprandial lipid levels with increase of eicosapentaenoic and docosahexaenoic acid contents in rat hepatic membrane. Lipids 36, $1331-1336$

34. Abedin L, Lien EL, Vingrys AJ, et al. (1999) The effects of dietary $\alpha$-linolenic acid compared with docosahexaenoic acid on brain, retina, liver, and heart in the guinea pig. Lipids $\mathbf{3 4}$, $475-482$.

35. Li D, Weisinger HS, Weisinger RS, et al. (2006) Omega 6 to omega 3 fatty acid imbalance early in life leads to persistent reductions in DHA levels in glycerophospholipids in 
rat hypothalamus even after long-term omega 3 fatty acid repletion. Prostaglandins Leukot Essent Fatty Acids 74, 391-399.

36. Stender S \& Dyerberg J (2004) Influence of trans fatty acids on health. Ann Nutr Metab 48, 61-66.

37. Mahfouz MM, Smith TL \& Kummerow FA (1984) Effect of dietary fats on desaturase activities and the biosynthesis of fatty acids in rat-liver microsomes. Lipids 19, 214-222.

38. Hornstra G (2000) Essential fatty acids in mothers and their neonates. Am J Clin Nutr 71, 1262S-1269S.

39. Larqué E, Pérez-Llamas F, Puerta V, et al. (2000) Dietary trans fatty acids affect docosahexaenoic acid concentrations in plasma and liver but not brain of pregnant and fetal rats. Pediatr Res $\mathbf{4 7}$, $278-283$.

40. Salmerón J, Hu FB, Manson JE, et al. (2001) Dietary fat intake and risk of type 2 diabetes in women. Am J Clin Nutr 73, 1019-1026.

41. Kavanagh K, Jones KL, Sawyer J, et al. (2007) Trans fat diet induces abdominal obesity and changes in insulin sensitivity in monkeys. Obesity 15, 1675-1684.
42. Smith U (2002) Impaired ('diabetic') insulin signaling and action occur in fat cells long before glucose intolerance - is insulin resistance initiated in the adipose tissue? Int $J$ Obes Relat Metab Disord 26, 897-904.

43. Pittas AG, Joseph NA \& Greenberg AS (2004) Adipocytokines and insulin resistance. J Clin Endocrinol Metab 89, 447-452.

44. Saravanan N, Haseeb A, Ehtesham NZ, et al. (2005) Differential effects of dietary saturated and trans-fatty acids on expression of genes associated with insulin sensitivity in rat adipose tissue. Eur J Endocrinol 153, 159-165.

45. Albuquerque KT, Sardinha FL, Telles MM, et al. (2006) Intake of trans fatty acid-rich hydrogenated fat during pregnancy and lactation inhibits the hypophagic effect of central insulin in the adult offspring. Nutrition 22, 820-829.

46. Sandra A, Fyler DJ \& Marshall SJ (1984) Effects of lipids on the transport activity of the reconstituted glucose transport system from rat adipocyte. Biochim Biophys Acta 778, 511-515.

47. Spector AA \& Yorek MA (1985) Membrane lipid composition and cellular function. J Lipid Res 26, 1015-1035. 